\title{
Role of probiotics and vitamins in maintaining a healthy gut microbiome: recent advances
}

Bani Tamber Aeri

Professor, Department of Food and Nutrition, Institute of Home Economics, University of Delhi.

\begin{tabular}{|c|c|c|c|c|c|c|c|c|}
\hline Abstract & Introduction & Methodology & Results & Conclusion & References & Citation & \multicolumn{2}{|c|}{ Tables / Figures } \\
\hline \multicolumn{9}{|c|}{ Corresponding Author } \\
\hline \multicolumn{8}{|c|}{$\begin{array}{l}\text { Dr Bani Tamber Aeri, Assistant Professor, Department of Food and Nutrition, Institute of Home Economics, } \\
\text { University of Delhi - } 110016 \\
\text { E Mail ID: } \underline{\text { bani.aeri@ihe.du.ac.in }}\end{array}$} & 回语四 \\
\hline
\end{tabular}

\section{Citation}

Aeri BT. Role of probiotics and vitamins in maintaining a healthy gut microbiome: recent advances. Indian J Comm Health. 2021;33(3):543-546. https://doi.org/10.47203/IJCH.2021.v33i03.027

Source of Funding: Nil Conflict of Interest: None declared

\section{Article Cycle}

Received: 06/09/2021; Revision: 16/09/2021; Accepted: 26/09/2021; Published: 30/09/2021

This work is licensed under a Creative Commons Attribution 4.0 International License.

\section{Abstract}

While the concept of probiotics is not new, science based approaches to developing targeted probiotic products are a renewed interest. Probiotics and the microbiome is now being considered as having a blueprint of good health, unique to every human. Along with the well-known and established health benefits like reduced antibiotic associated diarrhoea and colic symptoms, eczema, necrotising enterocolitis, acute paediatric infections like diahorrea and upper respiratory tract infections; probiotics are now considered as beneficial for maintaining mental well-being as well. This new branch of disease management is now termed as Nutritional neuroscience and these beneficial gut bacteria are referred to as psychobiotics. It is important to identify the specific strains of probiotics and characterize them and conduct randomised controlled trials to establish these benefits. With emerging data related to role of vitamins in modulating the gut microbiome, combining pre and probiotics with micronutrients is likely to result in powerful functional foods boosting host immunity.

\section{Keywords}

Anxiety; Depression; Gut-Brain Axis; Microbiome; Probiotics; Psychobiotics

\section{Introduction}

The term 'probiotic' was defined in 2001 by FAO/WHO as "live microorganisms which when administered in adequate amounts confer a health benefit on the host".(1) In 2013, the International Scientific Association for Probiotics and Prebiotics (ISAPP) defined probiotics as "Live microorganisms that, when administered in adequate amounts, confer a health benefit on the host." (2)

The microbiome which was earlier thought to only digest the insoluble polysaccharides, performs several other functions and is critical in maintenance of host health and prevention of diseases. The Gut microbiome consists of 1014 bacteria which could belong to $800-1000$ species most common being the Firmicutes, Bacteroidetes, Protinobacteria and Actinobacteria. (3). By the end of the first year of life, the microbial profile is distinct for each infant; by the age of 2.5 years, the microbiota fully resembles the microbiota of an adult in terms of composition. (4)
Factors known to influence gut colonization include gestational age, mode of delivery (vaginal birth vs assisted delivery), diet (breast milk vs formula), level of sanitation, and exposure to antibiotics. (5) Though the microbiome can be modulated by factors like physical activity, a wholesome diet is the key determinant of the gut microbiome and targeted nutrition may influence the gut microbiome positively. Although there is a lot of renewed interest in nutrition scientists about fermented foods but they are distinct from probiotic foods. (6)

\section{Microbiome and Health}

Probiotics have been associated with three core benefits which have been supported with high quality metaanalysis and RCT's: these being i) supporting a healthy gut microbiota ii) supporting a healthy digestive tract iii) supporting a healthy immune system.

Some of these core benefits have also been previously discussed: (7)

- Incidence and duration of antibiotic associated diarrhoea 
- Colic symptoms in breastfed babies

- Occurrences of atopic issues such as eczema in infants

- Necrotising enterocolitis in preterm infants

- Reduce symptoms of lactose maldigestion

- Acute pediatric infectious diahorrea

- $\quad$ Risk and duration of upper respiratory tract infections

- Probiotics and eradication of H Pylori: A recent metaanalysis of 40 studies with over 8000 participants which was conducted to evaluate the effectiveness and safety of probiotics in facilitating the eradication of $\mathrm{H}$ pylori concluded that Probiotics improved the eradication rate and reduced side effects when added to the treatments designed to eradicate $\mathrm{H}$ pylori. The eradication effects were better when probiotics were used before, throughout as well as up to 2 weeks after the treatment. (8)

- A systematic review based on 23 randomized controlled trials (RCT's) involving 6269 children to investigate the effect of probiotic consumption on respiratory tract infections (RTI's) in children indicated that probiotic consumption significantly decreased the number of subjects having at least one RTI episode. They even had a fewer number of days of absenteeism from day care/school; clearly indicating that probiotic consumption appears to be a feasible way to decrease the incidence of RTI in children. (9) In fact, recent evidence from six RCTs conducted on 1551 adults also provides support that probiotics have potential efficacy for preventing URTI episodes in adults as well. (10)

- Probiotics have also been used as treatment of certain food allergies like cow's milk and peanut allergy in pediatric populations. (11)

- Probiotics and antibiotics: Probiotic consumption may be a replacement for antibiotics as patients and clinicians may manage self-limited illnesses. A metaanalysis of 17 studies conducted on infants and children with a primary aim of preventing acute upper respiratory tract infections, acute lower digestive tract infections and otitis media concluded that subjects who received probiotic supplementation (ranging from 4 days to 9 months) had a lower risk of being prescribed antibiotics, relative to those who received placebo. (12) The effect of probiotics depend on host physiology and individual microbiome composition.

\section{Gut microbiome and brain function}

The gut microbiota is now known to communicate with the brain. (13) It was earlier thought that cells in the gut transduce sensory information through the paracrine action hormones. However, recent research has indicated that enteroendocrine cells present in the gut are able to communicate with the brain (vagal neurons) by using glutamate as a neurotransmitter. These synaptically connected cells have been referred to as neuropod cells.
(14) The gut microbiota influences the bidirectional communication between the central and enteric nervous system linking the brain with peripheral intestinal functions. This is done by the products of the microbiota, the short chain fatty acids and the direct influence of the microbiome in the formation of neurotransmitters, the cytokine response and the Gut Brain Axis (GBA). (15)

Short chain fatty acids produced by the microbiome can act as the trigger. Ninety percent of Serotonin (the feel good hormone) is produced by the indigenous bacteria of the gut microbiome. (16) Gut microbiota is also perhaps associated with the brain development and disorders. This was proven in animal models and data indicated that germ free mice had defective brain function and altered behaviour. (17) In fact certain mental disorders like epilepsy, stress disorders, autism spectrum disorders and depression have shown improvement if the therapy included management of dysbiosis of the gut. Even patients of Parkinson's disease have shown to suffer from GI disorders. A compound alpha- synuclein which has been found in the degenerating neurons of such patients has also been found in the neurons of the enteric nervous system. It is possible that microbial metabolites may be involved in the formation of alpha synuclein and this travels along the Vagus nerve to the brain. (18)

This new branch of disease management is now termed as Nutritional Neuroscience and these beneficial gut bacteria are referred to as psychobiotics. (19) There is growing evidence indicating that changes in the composition of the gut microbiota are related to mental health including anxiety and depression. The brain neurotransmitter modulation by gut microbiota is indispensable to the physiopathology of anxiety and depression. Gut microbiota provides a novel way to alter neurotransmitter modulation in the brain and treat gut-brain axis diseases. Dysbiosis of the gut microbiota has been indicated in the late onset autism spectrum disorders accompanied by GI symptoms like distention, abdominal discomfort and changes in bowel habits. An Indian study on autistic children showed prominent dysbiosis in the gut microbiome of ASD children, with higher relative abundances of families Lactobacillaceae, Bifidobacteraceae, and Veillonellaceae. (20)

Depressive symptoms are also associated with irritable bowel syndrome and presence of pro inflammatory cytokines favours the conversion of tryptophan into kyneurinine which results in depression. (21) The gut microbiota is proposed to contribute to blood-brain barrier disruption and the pathogenesis of neurodegenerative diseases. (22) Hence the 'Gut Brain' not only completes its local function but also regulates the human behaviour and cognition, similar to the brain.

\section{Next generation Probiotics}

These are the probiotics which are isolated from the gut itself based on metagenomic analysis comparing control 
vs disease situations. The probiotics currently available to consumers are generally drawn from a narrow range of organisms. Knowledge of the gut microbiome is now making it possible to isolate the gut microbiota and use them to treat disease conditions like diabetes or heart disease. Developments in affordable complete genome sequencing and powerful cultivation methods have allowed isolation and characterisation of a new range of microorganisms from human microbiomes with potential health benefits and the opportunity to be developed as next-generation probiotics. (23) Various bacteria, such as Roseburia intestinalis, Faecalibacterium prausnitzii, Eubacterium spp., Bacteroides spp. and Akkermansia muciniphila, have been isolated from the human gut with growing interest in their probiotic potential For this reason, and because their development is likely to be more amenable to a pharmaceutical than a food delivery route, these organisms are often operationally referred to as next-generation probiotics, a concept that overlaps with the emerging concept of live biotherapeutic products. The latter is a class of organisms developed exclusively for pharmaceutical application. (23) Although the next generation probiotics have advantage of being a major component of the gut microbiome and thus would aid in the formation of bioactives; there are certain challenges associated with them. The industrial production being one, since these microbes need complex growth media and anaerobic conditions. Along with this the regulatory processes may need looking into.

\section{Safety considerations}

It is important to identify the specific strains of probiotics and characterize them. Clinical studies involving probiotics have limitations (of numbers and diverse populations which may not be representatives of a particular community), extrapolation from models like the artificial gut system too has limitations. Probiotics are complex mixtures and they represent quality assurance challenges because different preparations have different mechanisms of action.

Antimicrobial resistance (AMR) is a global threat gaining more and more practical significance every year. The main determinants of AMR are the antimicrobial resistance genes (ARGs). Since bacteria can share genetic components via horizontal gene transfer, even nonpathogenic bacteria may provide ARG to any pathogens which they become physically close to (e.g. in the human gut). Fermented food naturally contain bacteria in high amounts and during the fermentation process, the ARG content of foods can grow due to bacterial multiplication. Thus, the challenge is to monitor and select the starting culture strains of fermented foods in order to decrease the intake of ARGs via foods. (24)

\section{Vitamins and Gut Microbiome}

While modulation of the gut microbiota by pro-and prebiotics is already well established, the effects of amino acids, certain lipids, enzymes, minerals, and vitamins is less clear. Traditionally, these nutrients were not thought to impact the gut microbiome because they do not reach the distal gastrointestinal tract but are absorbed efficiently in the proximal small intestine. Interestingly, data now indicates that vitamins when overdosed or delivered directly to the colon, can modulate the gut microbiome. (25) Moreover, studies in animals and humans show that microbes can synthesize vitamin $C$ and $\mathrm{K}$ and the $\mathrm{B}$ group vitamins themselves and that under certain disease conditions like inflammatory bowel disease or even malnutrition, the intrinsic gut microbiome based vitamin production is decreased. Currently, the possibility of regulating gut microbiota to reverse these perturbations by developing novel therapeutic and preventive strategies is being extensively investigated. A recent clinical study including healthy volunteers investigated the effects of colon-delivered vitamins A, B2, $C, D$, and $E$ on the gut microbiota. The results indicated that vitamins C, B2, and D can modulate the human gut microbiome in terms of metabolic activity and bacterial composition in varying degrees. Vitamin C significantly increased microbial alpha diversity and fecal butyrate and propionate concentrations as well as microbial evenness when compared with placebo. Follow-up studies investigating targeted delivery of vitamins to the colon may help clarify the clinical significance of this novel concept for treating and preventing dysbiotic microbiotarelated human diseases. $(26,27)$ Combining pre and probiotics with micronutrients is likely to result in powerful functional foods boosting host immunity.

\section{Conclusion}

There is a rapidly expanding need for probiotics for immune benefit. We now have the opportunity and the know-how to study various strains which include target specific strain selections. Probiotic rich fermented food products have a huge potential once they overcome the regulatory, industrial and quality assurance challenges. Probiotics may contribute hugely to global health in particular their immune response against virus and other disease causing organisms along with mental health. Vitamins too may hold the key to healthy gut microbiome. Combining pre and probiotics with micronutrients is likely to result in powerful functional foods boosting host immunity.

\section{Recommendation}

Probiotics and prebiotics along with vitamins have huge potential in addressing gut health which will impact both physical and mental well being.

\section{Acknowledgement}

The paper is based on inputs from Prof. Patricia Conway, Adjunct Professor, University of New South Wales, Australia and Visiting Professor, Nanyang Technological University, Singapore; Robert E. Steinert, PhD, EMEA 
Principal Scientist, DSM Nutritional Products, R\&D Human Nutrition and Health, Basel, Switzerland and $\mathrm{Dr}$ B.Sesikeran MD Chairman, Knowledge Center on Functional foods, Immunity and Gut Microbiome (K-FFIG), Former Director, National Institute of Nutrition (ICMR) during the International Life Sciences Institute (ILSI), India and K-FIG's webinar on 'Recent knowledge on nutrients health and immunity' held on 26th Feb, 2021

\section{References}

1. Food and Agriculture Organisation. Available at: http://www.fao.org/3/a0512e/a0512e.pdf (Accessed on 25/09/2021)

2. International Scientific Association for probiotics and Prebiotics. Available at: https://isappscience.org/forscientists/resources/probiotics (Accessed on 25/09/2021)

3. Quigley EM. Gut bacteria in health and disease. Gastroenterology \& hepatology. 2013; 9(9): 560-69.

4. Palmer C, Bik EM, DiGiulio DB, Relman DA, Brown PO. Development of the human infant intestinal microbiota. PLoS Biol. 2007;5(7):e177.

5. Fouhy F, Ross RP, Fitzgerald GF, Stanton C, Cotter PD. Composition of the early intestinal microbiota: knowledge, knowledge gaps and the use of high-throughput sequencing to address these gaps. Gut Microbes. 2012;3(3):203-20.

6. Marco ML, Sanders ME, Gänzle M, Arrieta MC, Cotter PD, Vuyst LC, et al. The International Scientific Association for Probiotics and Prebiotics (ISAPP) consensus statement on fermented foods. Nat Rev Gastroenterol Hepatol. 2021; 18: 196-208. https://doi.org/10.1038/s41575-020-00390-5

7. ILSI, India Status Paper on Probiotics, 2019 http://ilsiindia.org/PDF/Status_Paper_On_Role_of_Probiotics_In_Promotin g_Healthy Microbiome For Health_And Immunity/Status Paper On Role of Probiotics In Promoting Healthy Microbiome For Health And Immunity.pdf (Accessed on 25/09/2021)

8. Shi X, Zhang J, Mo L, Shi J, Qin M, \& Huang X. Efficacy and safety of probiotics in eradicating Helicobacter pylori: A network metaanalysis. Medicine. 2019; 98(15), e15180. https://doi.org/10.1097/MD.0000000000015180

9. Wang $Y$, Li X, Ge T, Xiao, Y, Liao Y, Cui $Y$ et al. Probiotics for prevention and treatment of respiratory tract infections in children, Medicine,2016; 95(31): e4509 doi: 10.1097/MD.0000000000004509

10. Laodong L, KangKang $H$, Qixiang S, Huan X, Lejin L, Moyu M, Chaoqian L. Probiotics for Preventing Upper Respiratory Tract Infections in Adults: A Systematic Review and Meta-Analysis of Randomized Controlled Trials. Evidence-Based Complementary and Alternative Medicine, 2020; vol. 2020, Article ID 8734140, 8 pages. https://doi.org/10.1155/2020/8734140

11. Tan-Lim C and Esteban-Ipac N. Probiotics as treatment for food allergies among pediatric patients: a meta-analysis. The World Allergy Organization journal. 2018; 11(1): 25. https://doi.org/10.1186/s40413-018-0204-5

12. King, Tancredi D, Lenoir-Wijnkoop I, Gould K, Vann H, Connors G et al. Does probiotic consumption reduce antibiotic utilization for common acute infections? A systematic review and meta-analysis.
[Role of probiotics...] | Aeri Bani T et al European journal of public health, 2019; 29(3): 494-499. https://doi.org/10.1093/eurpub/cky185

13. Mohajeri MH, La Fata G, Steinert RE, Weber P. Relationship between the gut microbiome and brain function. Nutr Rev, 2018;76(7):481-496. doi: 10.1093/nutrit/nuy009.

14. Kalberer MM, Buchanan KL, Klien ME, Barth BB, Montoya MM, Shen X, Bohorquez DV. A gut-brain neural circuit for nutrient sensory transduction Science, 2018; 361(6408): eaat5236 DOI: 10.1126/science.aat5236

15. Parker A, Fonseca S and Carding SR. Gut microbes and metabolites as modulators of blood-brain barrier integrity and brain health. Gut Microbes, 2020; 11(2): 135-157, DOI: 10.1080/19490976.2019.1638722

16. Yano JM, Yu K, Donaldson GP, Shastri GG, Ann P, Ma L et al. Indigenous bacteria from the gut microbiota regulate host serotonin biosynthesis. Cell, 2015; 161(2): 264-276. https://doi.org/10.1016/i.cell.2015.02.047

17. Carabotti M, Scirocco A, Maselli M A, and Severi C. The gut-brain axis: interactions between enteric microbiota, central and enteric nervous systems. Annals of gastroenterology, 2015; 28(2), 203209.

18. Gazerani P. Probiotics for Parkinson's Disease. International Journal of Molecular Sciences. 2019; 20(17):4121. https://doi.org/10.3390/ijms20174121

19. Butler MI, Sandhu K, Cryan JF, Dinan TG. From isoniazid to psychobiotics: the gut microbiome as a new antidepressant target. $\mathrm{Br} J$ Hosp Med (Lond). 2019; 80(3):139-145. doi: 10.12968/hmed.2019.80.3.139.PMID: 30860919

20. Pulikkan J, Maji A, Dhakan DB, Saxena R, Mohan B, Anto MM et al. Gut Microbial Dysbiosis in Indian Children with Autism Spectrum Disorders. Microbial ecology, 2018; 76(4), 1102-1114. https://doi.org/10.1007/s00248-018-1176-2

21. Huang $\mathrm{F}$ and $\mathrm{Wu} \mathrm{X}$. Brain Neurotransmitter Modulation by Gut Microbiota in Anxiety and Depression. Front Cell Dev Biol, 11 March 2021 https://doi.org/10.3389/fcell.2021.649103

22. Chen Y, Xu J, and Chen Y. Regulation of Neurotransmitters by the Gut Microbiota and Effects on Cognition in Neurological Disorders. Nutrients, 2021; 13(6), 2099. https://doi.org/10.3390/nu13062099

23. O'Toole P, Marchesi J and Hill C. Next-generation probiotics: the spectrum from probiotics to live biotherapeutics. Nat Microbiol, 2017 ; 2, 17057 https://doi.org/10.1038/nmicrobiol.2017.57

24. Tóth AG, Csabai I, Maróti G, Jerzsele A, Dubecz A, Patai AV et al. A glimpse of antimicrobial resistance gene diversity in kefir and yoghurt. Sci Rep , 2021; 10, 22458. https://doi.org/10.1038/s41598-020-80444-5 (Accessed on 25/09/2021)

25. Steinert RE, Lee YK, Sybesma W. Vitamins for the Gut Microbiome. Trends Mol Med. 2020;26(2):137-140. doi 10.1016/j.molmed.2019.11.005. Epub 2019 Dec 17.

26. Pham VT, Calatayud M, Rotsaert C, Seifert N, Richard N, Van den Abbeele $P$, Marzorati M, Steinert RE. Antioxidant Vitamins and Prebiotic FOS and XOS Differentially Shift Microbiota Composition and Function and Improve Intestinal Epithelial Barrier In Vitro. Nutrients. 2021;13(4):1125. doi: 10.3390/nu13041125.

27. Pham VT, Fehlbaum S, Seifert N, Richard N, Bruins MJ, Sybesma W, Rehman A, Steinert RE. Effects of colon-targeted vitamins on the composition and metabolic activity of the human gut microbiomea pilot study. Gut Microbes. 2021;13(1):1-20. doi: 10.1080/19490976.2021.1875774. 